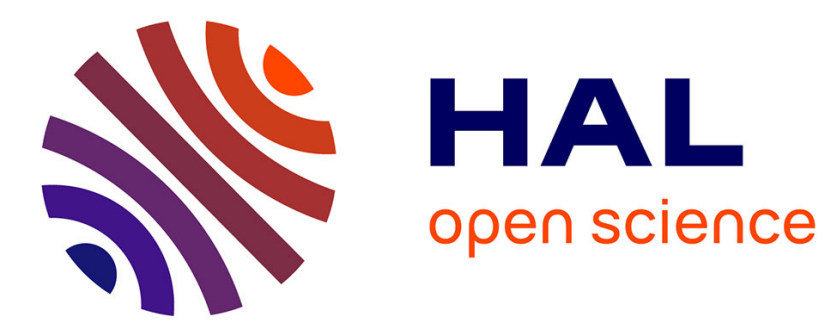

\title{
Small angle neutron scattering (SANS) under non-equilibrium conditions
}

\author{
R.C. Oberthür
}

\section{To cite this version:}

R.C. Oberthür. Small angle neutron scattering (SANS) under non-equilibrium conditions. Revue de Physique Appliquée, 1984, 19 (9), pp.663-670. 10.1051/rphysap:01984001909066300 . jpa-00245235

\section{HAL Id: jpa-00245235 https://hal.science/jpa-00245235}

Submitted on 1 Jan 1984

HAL is a multi-disciplinary open access archive for the deposit and dissemination of scientific research documents, whether they are published or not. The documents may come from teaching and research institutions in France or abroad, or from public or private research centers.
L'archive ouverte pluridisciplinaire HAL, est destinée au dépôt et à la diffusion de documents scientifiques de niveau recherche, publiés ou non, émanant des établissements d'enseignement et de recherche français ou étrangers, des laboratoires publics ou privés. 


\title{
Small angle neutron scattering (SANS) under non-equilibrium conditions
}

\author{
R. C. Oberthür \\ Institut Laue-Langevin, 156X, 38042 Grenoble Cedex, France
}

\begin{abstract}
Résumé. - L'utilisation de la diffusion des neutrons aux petits angles(DNPA) pour l'étude des systèmes hors d'équilibre thermodynamique est illustrée par trois types d'expériences dans le domaine de la recherche sur les polymères : - la relaxation d'un système à partir d'un état hors d'équilibre vers l'équilibre,

- l'installation cyclique ou répétitive d'une série d'états hors d'équilibre dans un système,

- l'état stationnaire, maintenu par une dissipation constante d'énergie dans le système.

Les temps caractéristiques accessibles dans ces expériences par DNPA sont comparés aux temps obtenus dans les expériences de diffusion quasi élastique des neutrons et de la lumière, qui fournissent l'information sur la dynamique des fluctuations à l'état d'équilibre thermodynamique. Les limites de la DNPA pour l'obtention des temps de relaxation aux différentes échelles de longueur dans des systèmes hors d'équilibre sont mises en évidence et comparées aux limites propres à la diffusion quasi élastique des neutrons et de la lumière.
\end{abstract}

\begin{abstract}
The use of small angle neutron scattering (SANS) for the study of systems under non-equilibrium conditions is illustrated by three types of experiments in the field of polymer research :

- the relaxation of a system from an initial non-equilibrium state towards equilibrium,

- the cyclic or repetitive installation of a series of non-equilibrium states in a system,

- the steady non-equilibrium state maintained by a constant dissipation of energy within the system.

Characteristic times obtained in these experiments with SANS are compared with the times obtained from quasielastic neutron and light scattering, which yield information about the equilibrium dynamics of the system. The limits of SANS applied to non-equilibrium systems for the measurement of relaxation times at different length scales are shown and compared to the limits of quasielastic neutron and light scattering.
\end{abstract}

\section{Introduction.}

The essentials of non-equilibrium sample environments may be best defined with respect to thermodynamic equilibrium. The criterium of equilibrium for an isolated system is given by a maximum of entropy $S$, corresponding to zero production of entropy :

$$
\begin{aligned}
S & =S_{\max } \\
\mathrm{d} S & =0 .
\end{aligned}
$$

Equilibrium conditions include the influence of temperature, pressure, and chemical composition, as well as the influence of constant external fields. These external fields may be a constant electrostatic or magnetic field as well as an externally imposed stress. Measurements of a sample at thermodynamic equilibrium provide informations on time independent properties of matter.

In all real systems, however, fluctuations about the so defined equilibrium state occur. From statistical thermodynamics the probability of deviations from the most probable equilibrium state are obtained (cf. [1-3]). These fluctuations may result in a temporal decrease or increase of entropy, but with a time averaged entropy equal to $S_{\max }$ and hence. no net entropy production (Fig. 1a).

Measurements of such fluctuations provide information about the equilibrium dynamics of a given system, as expressed in a characteristic correlation function of the fluctuations (Fig. 1b) and a characteristic relaxation time $\tau$. Nevertheless, the fluctuations about equilibrium are already a first step towards non-equilibrium since a large spontaneous deviation from equilibrium due to statistical fluctuations, albeit seldom, can a priori not be distinguished from an externally imposed non-equilibrium state.

The definitive step towards non-equilibrium consists in subjecting a system directly to non-equilibrium conditions. This can be done by mixing together 

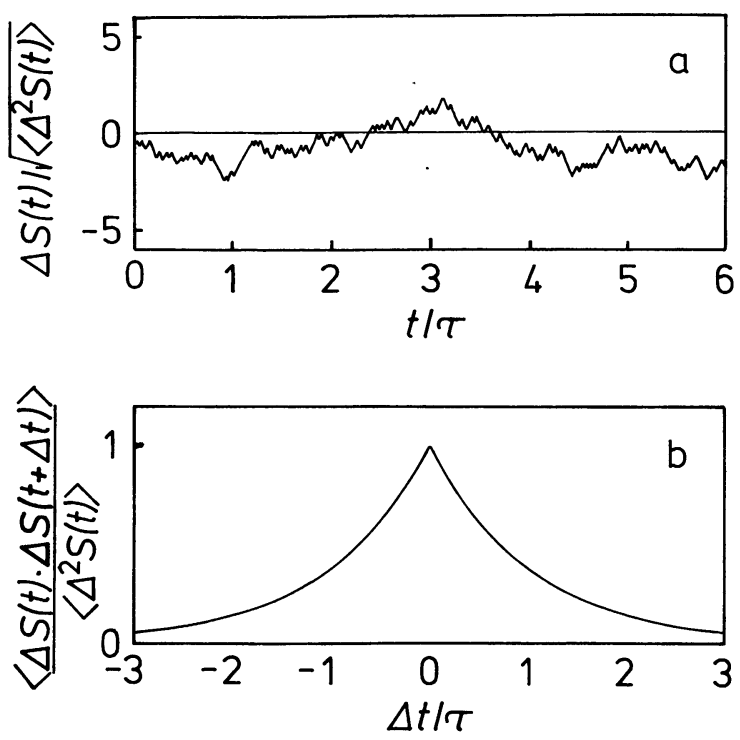

Fig. 1. - Fluctuations about an average thermodynamic quantity (a) and corresponding correlation function of the fluctuations (b). The correlation between the fluctuations is invariant with respect to time inversion (criterium for thermodynamic reversibility).

two substances, each at equilibrium, whereas the mixture is not, or by changing the external conditions like temperature, pressure or an external field (Fig. 2a), which suddenly places the system under non-equilibrium conditions. In such cases, the system tends to relax towards equilibrium at a given relaxation rate (Fig. 2b).

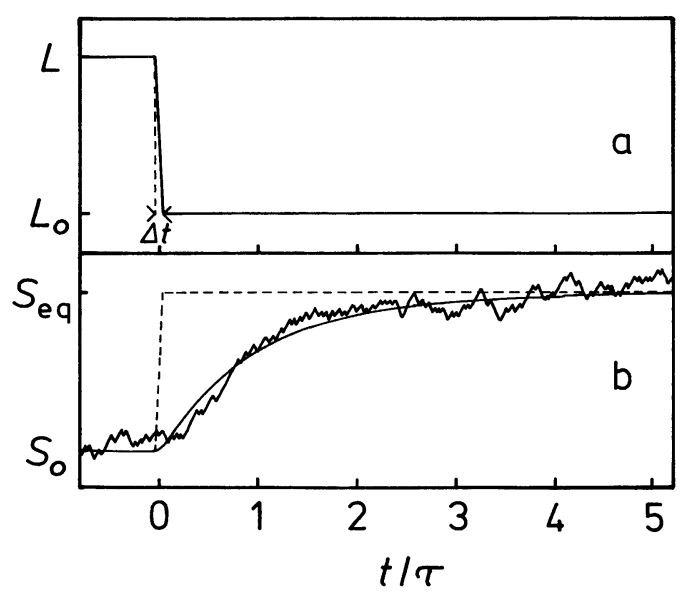

Fig. 2. - Relaxation of the thermodynamic quantity $S$ (b) as response to a sudden change in a thermodynamic variable $L$ (a), which places the system under non-equilibrium conditions.

This means that in contrast to equilibrium thermodynamics, non-equilibrium thermodynamics always deal with time dependent phenomena. Hence, all measurements of systems under non-equilibrium conditions always introduce a new dimension into the experimental data : the time. However, when equilibrium conditions are approached, the relaxation rates obtain- ed from non-equilibrium measurements should become identical to those already obtained from equilibrium dynamic measurements of the fluctuations about equilibrium (cf. Figs. 1 and 2).

A crucial parameter for the design of non-equilibrium experiments is the time $\Delta t$ required to install non-equilibrium conditions (cf. Fig. 2a). If $\Delta t$ is large with respect to the relaxation times of the system, then reversible changes occur, equilibrium conditions are maintained with a total recovery of the input energy. Hence, no energy is dissipated and no entropy is produced. Only if $\Delta t$ is comparable to or less than the relaxation times of the system, irreversible changes occur, and non-equilibrium conditions are installed, which then tend to relax towards equilibrium with energy dissipation and the production of entropy.

Three different types of experiments under nonequilibrium conditions will be considered in the following :

- kinetic or relaxation experiments. where from a given initial non-equilibrium state the system is allowed to approach equilibrium;

- cyclic experiments, where non-equilibrium conditions are imposed in a cyclic way, and

- steady state experiments. where a given nonequilibrium state is maintained by a constant dissipation of energy.

The study of the conformation of a polymer chain by small angle neutron scattering (SANS) is taken as a typical example to illustrate these three nonequilibrium conditions and the inherent limits of the SANS technique due to the required sample environment.

\section{Relevant characteristics and limits of neutron scat- tering.}

A major advantage of neutron scattering with respect to absorption spectroscopy is its potential information on space and time resolved properties of the sample to be investigated. Hence, in the particular case of non-equilibrium experiments relaxation times and even fluctuations around the non-equilibrium states could be obtained at different space scales.

The typical space scale for small angle neutron scattering (SANS) ranges from $\mathrm{nm}$ to $\mu \mathrm{m}$, whereas with neutron diffraction the $\mathrm{pm}$ to $\mathrm{nm}$ range is reached. In this latter domain atomic to molecular phenomena of matter with a typical time scale in the fs to ns range are predominant. This space/time domain is well covered by inelastic neutron scattering. SANS, however, reveals the shape of large molecules and atomic or molecular clusters with associated characteristic times for structural rearrangements easily ranging from $\mu$ s to days. This domain is only marginally covered by quasielastic neutron scattering (neutron spin echo (NSE)) due to its upper limit in time resolution, and by quasielastic light scattering (QELS) due to its upper limit in space resolution (Fig. 3). 


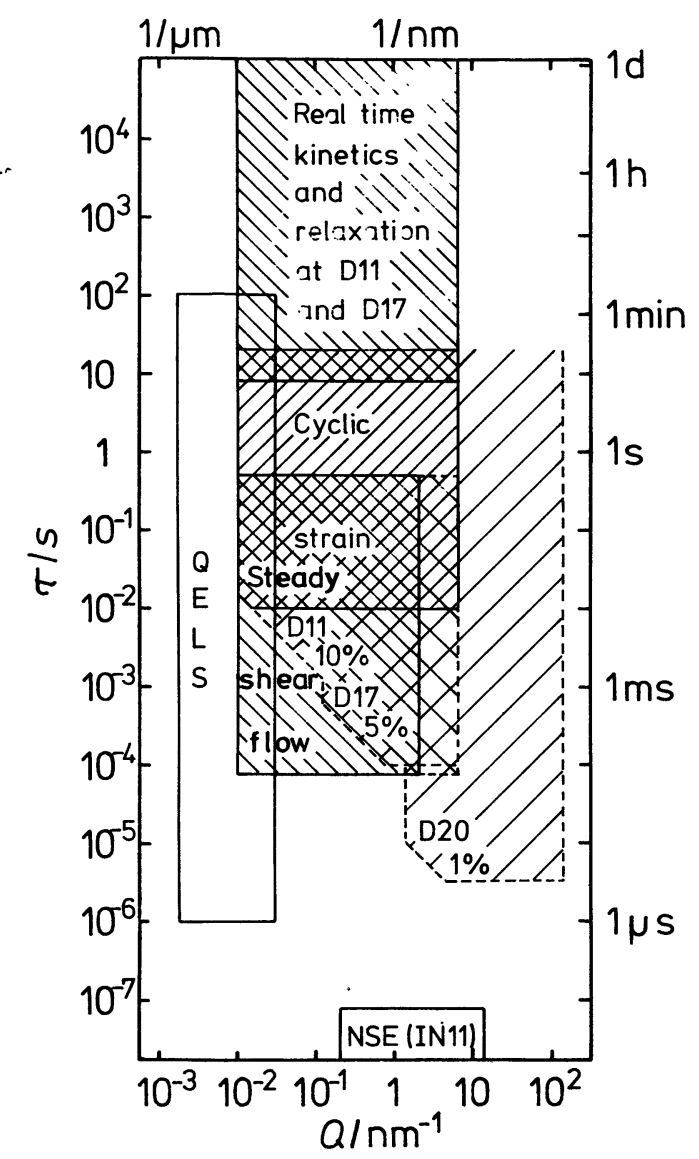

Fig. 3. - Comparison of the range of time and space resolution of neutron scattering from samples under non-equilibrium conditions with the corresponding range of quasielastic neutron scattering (neutron spin echo : NSE at IN11) and quasielastic light scattering (QELS).

On the other hand, the information to be obtained depends on the scattered intensity to be subdivided into space channels (scattering direction), correlation time channels (energy of scattered neutrons), and real time channels for the different non-equilibrium states. This offers a huge amount of simultaneous information about the sample, but requires also a huge amount of scattered neutrons to be subdivided according to the three different criteria. Thus, the first inherent limits for neutron scattering investigations come from the limited primary beam intensity $Y_{0}$ and the limited detector counting and selection rate for scattered neutrons, due to dead time losses and electronic problems of data handling. Energy resolution, which might be taken into account for experiments with pulsed neutron sources, is therefore neglected and only space and real time resolution is considered for experiments to be performed with the continuous neutron flux of a reactor.

A survey of instrumental parameters relevant for the design of non-equilibrium experiments under these conditions is given below :

\section{$Y_{0} \quad$ neutron primary beam intensity}

$v \quad$ neutron velocity

REVUE DE PHYSIQUE APPLIQUÉE. - T. 19, № 9, SEPTEMBRE 1984 $\lambda \quad$ neutron wavelength with $\lambda[\mathrm{nm}]=396 / v[\mathrm{~m} / \mathrm{s}]$

$\Delta \lambda / \lambda$ relative width of wavelength distribution

$\Delta A \quad$ surface of sample diaphragm

$D$ thickness of sample container and sample

$T$ transmission of sample container and sample

$\mathrm{d} \Sigma / \mathrm{d} \Omega$ scattering cross section of sample container and sample per unit volume

$\theta \quad$ scattering angle (between direction of primary beam and scattered neutron)

$L \quad$ sample to detector distance

$\Delta R \quad$ width of primary beam at the detector

$R \quad$ distance between detected neutron and centre of primary beam at the detector

$Y_{\max }$ maximum counting rate per unit detector surface area.

The intensity at the detector is related to these parameters by

$$
\begin{aligned}
Y(Q)=\frac{Y_{0}}{L^{2}} & \Delta A \cdot T_{\mathrm{c}} \cdot T_{\mathrm{s}} \times \\
\times & {\left[D_{\mathrm{c}} \cdot\left(\frac{\mathrm{d} \Sigma}{\mathrm{d} \Omega}\right)_{\mathrm{c}}(Q)+D_{\mathrm{s}} \cdot\left(\frac{\mathrm{d} \Sigma}{\mathrm{d} \Omega}\right)_{\mathrm{s}}(Q)\right] }
\end{aligned}
$$

where the subscripts (c) and (s) refer to container and sample and

$$
Q=\frac{4 \pi}{\lambda} \cdot \sin \frac{\theta}{2}
$$

is the momentum transfer during the scattering process and carries the information about the distances within the sample.

A particularly important limitation of neutron scattering with respect to real time experiments is the time resolution of the obtained scattering pattern. It is given by the time spread $\Delta t$ with which the neutron arrives at the detector when it has hit the sample at a given time $t_{0}$. $\Delta t$ is equal to the time $t=L / v$, necessary for the neutron to travel from the sample to the detector, and to the relative time spread $\Delta t / t$, which results from the relative velocity spread $\mathrm{d} v / v$ of the incoming neutrons :

$$
\Delta t=t \cdot \frac{\Delta t}{t}=\frac{L}{v} \cdot \frac{\Delta v}{v} .
$$

The neutron velocity is related to the inverse of the neutron wavelength and hence

$$
\Delta t[\mathrm{~ms}]=2.5 \cdot \frac{\Delta \lambda}{\lambda} \cdot \lambda[\mathrm{nm}] \cdot L[\mathrm{~m}]
$$

The scattering vector $Q$ at small scattering angles is reduced to

$$
Q=\frac{2 \pi}{\lambda} \cdot \frac{R}{L}
$$


and thence in terms of $Q$ the time resolution reads

$$
\Delta t[\mathrm{~ms}]=15.7 \cdot \frac{\Delta \lambda}{\lambda} \cdot \frac{R[\mathrm{~m}]}{Q\left[\mathrm{~nm}^{-1}\right]} .
$$

According to this equation the crucial parameters for a good time resolution at a given $Q$ are the wavelength spread of the primary beam and the width of the primary beam at the detector, which determines the minimum value of $R$. Since the width of the primary beam is in the order of $4 \mathrm{~cm}$, a minimum value for $R=0.1 \mathrm{~m}$ was chosen for convenience. The limits so obtained for $\Delta t$ as a function of $Q$ are given in figure 3 for D11 with its $10 \%$ selector, for D17 with its $5 \%$ selector and for the new instrument D20, which is especially conceived for real time experiments, with a $1 \%$ monochromator [4].

\section{Experimental examples.}

3. 1 EQUILIBRIUM EXPERIMENTS. - The essential prerequisite for a sample to be measured under nonequilibrium conditions is a thorough understanding of its equilibrium scattering behaviour. Weak scatterers can readily be ruled out, since they would not supply enough neutrons to be subdivided into the various space and time channels. The second crucial requirement for experiments under nonequilibrium conditions is a sufficient difference $(>5 \%)$ in the scattered intensity at a given $Q$ in the different thermodynamic equilibrium states, between which the sample has to relax.

3.1.1 Static experiments. - An illustrative example for SANS measurements of a sample at different states of thermodynamic equilibrium, which can later be compared to a non-equilibrium experiment is a polydimethylsiloxane (PDMS) rubber under stress [5]. The stretched macroscopic network of cross-linked PDMS molecules hinders the individual elongated end-cross-linked chain molecules of molar mass $M=10^{4} \mathrm{~g} /$ mole, $20 \%$ of which are deuteriumlabelled (see insert in Fig. 4), relaxing back to their conformation in the unstretched state (cf. section 3.2). A new elongated chain conformation is adopted which, however, does not follow the variation in the outer dimensions of the stretched sample (affine in Fig. 4).

Based on the idea that neighbouring network points are not necessarily interconnected through one chain segment (cf. insert in Fig. 4) and on the fact that between the network points the coil molecules are in a liquid state (far above the glass transition temperature $T_{\mathrm{g}}=-120^{\circ} \mathrm{C}$ ) with appreciable mobility, the model of a phantom network has been developed [6], which explains the variation with elongation of the mean square radius both parallel and perpendicular to the strain with fair accuracy.

It follows that the variation of the scattering behaviour of the labelled segment obtained with changing

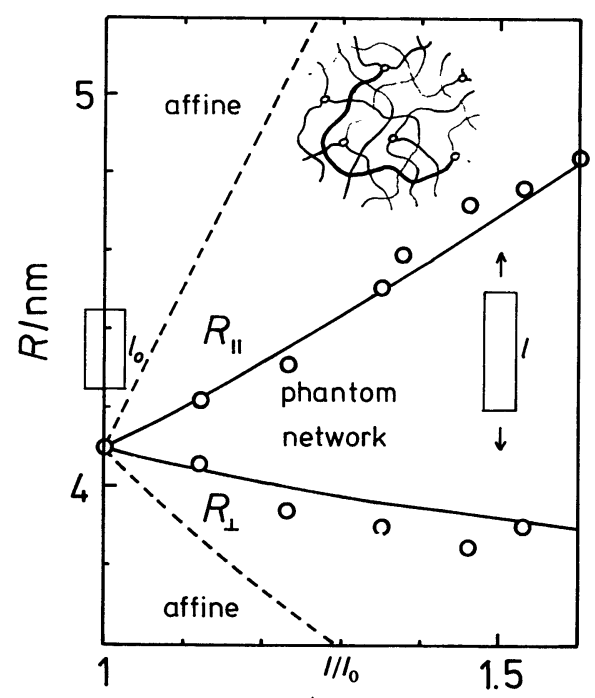

Fig. 4. - Variation of a statistical molecular parameter, the root of the mean square radius $R$ of a labelled macromolecular link in a rubbery PDMS network with a changing thermodynamic variable, the elongation $l / l_{0}$ of the sample. $(/ /)$ and $(\perp)$ denote the direction parallel and perpendicular to the strain [5], and the curves represent the calculated behaviour for an affine coil deformation and a deformed "phantom network".

external conditions is just good enough to make it a candidate for a non-equilibrium experiment (see 3.3).

3.1.2 Dynamic experiments. - Static experiments at equilibrium can already give valuable information about the scattered intensity, the space scale of the molecular parameters to be studied, and about the differences in the scattering behaviour with changing external conditions. Dynamic experiments at equilibrium will give the supplementary information about the time scale at which a system is ready to change from one thermodynamic state to another.

Quasielastic neutron and light scattering experiments with polymers measure the correlation function of the conformational fluctuation of the coil molecule at a given length scale. Hence, neutron scattering monitors the segmental mobility of a polymer chain in the $\mathrm{nm}$ and $\mathrm{ns}$ region, whereas light scattering reflects translational diffusion of the whole coil molecule and, only for large coils, shows the slowest Rouse mode of intersegmental diffusion (" breathing of the whole coil $")$ in the $\mu \mathrm{m}$ and $\mathrm{ms}$ range. Together with theoretical predictions for the first cumulant of the time correlation function [9] a rough, but complete picture about the $Q$-dependent dynamics of a single polystyrene chain in dilute solution emerges (Fig. 5).

A controlled variation of the slowest Rouse mode based on experimental data by variation of the molar mass of the polymer and the solvent viscosity can now be envisaged (circles in Fig. 5). This makes it possible to shift a space/time behaviour into the time domain of SANS under non-equilibrium conditions 
(arrows in Fig. 5), and hence to make an accurate plan for the envisaged experiment.

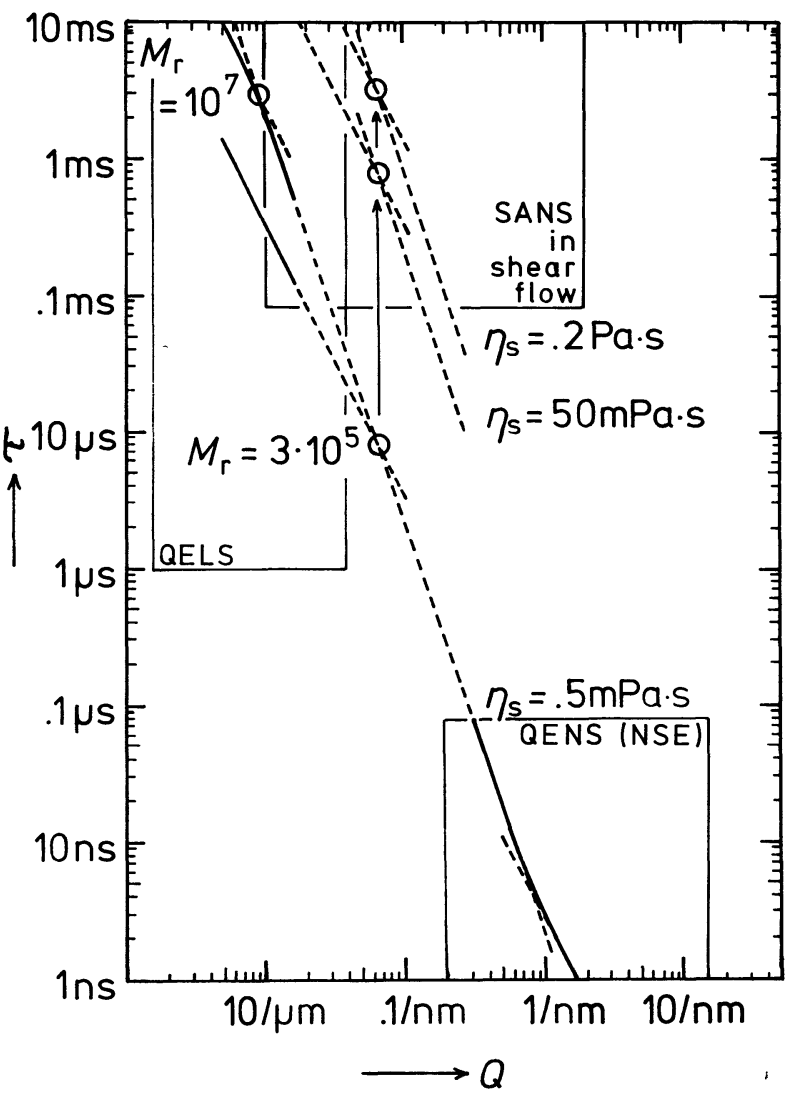

Fig. 5. - Relaxation times present in a dissolved polystyrene molecule as a function of the distances encountered in and by the polymer molecule. Drawn lines : data obtained by QENS [7] and QELS [8]. The dotted line gives the extrapolation between both experimental data according to theory [9]. The circles correspond to the slowest Rouse mode of the coil caused by a "breathing " of the whole molecule at distances of the order of the mean square radius. Variation of the solvent viscosity changes the time scale but not necessarily the distance scale of the conformational fluctuations of the coil.

3. 2 KINETIC OR RELAXATION EXPERIMENTS. - A particularly elegant method for SANS studies of the relaxation of deformed polymer coils back to their equilibrium state consists in a freezing of the different intermediate non-equilibrium states and allows to use all the advantages of neutron scattering with samples in an ambient environment. This technique is illustrated with the example of the relaxation of stretched deuterated polystyrene (PS) with molar mass $M=32000 \mathrm{~g} / \mathrm{mole}$ in a protonated matrix with $M=1.6 \times 10^{6} \mathrm{~g} /$ mole [10].

The samples were stretched above the glass transition temperature of PS $T_{\mathrm{g}}=100^{\circ} \mathrm{C}$ in a constant velocity gradient $G_{\mathrm{L}}=0.189 / \mathrm{s}$ in a time interval $\Delta t=7 \mathrm{~s}$ up to 3 times their initial length. They were then allowed to relax for a given time $t$ at constant length and temperature and the attained non-equili- brium state was frozen by quenching the sample to a temperature below $T_{\mathrm{g}}$ in less than $10 \mathrm{~s}$. The obtained reduced mean square radii parallel and perpendicular to the stretch are plotted as a function of $t$ (Fig. 6). A relaxation time $\tau=77 \mathrm{~s}$ is obtained.

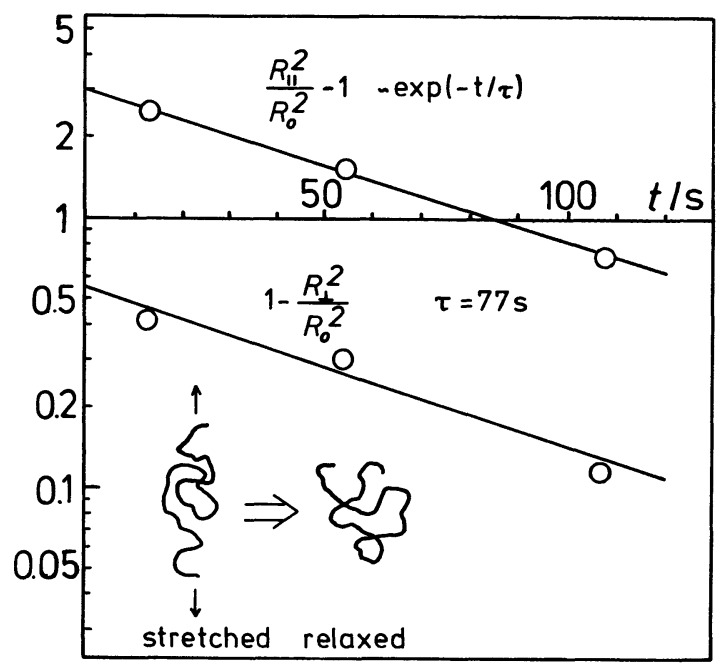

Fig. 6. - Relaxation of rapidly stretched PS molecules towards their equilibrium conformation as expressed in the variation with time of the mean square radius of the coil parallel $(/ /)$ and perpendicular $(\perp)$ to the bulk elongation with respect to the mean square radius at equilibrium $(O)$. Measurements at three different frozen non-equilibrium states [10].

This example contains all characteristics of a nonequilibrium experiment. The time interval $\Delta t$ to attain non-equilibrium conditions in conjuction with the reciprocal value of the velocity gradient as well as the quenching time to terminate the relaxation process are both small compared with the relaxation time $t$ of the deformed polymer coil, which means that the coil dimensions are initially deformed at the same rate as the bulk sample (affine deformation). However, when the flow gradient goes back to zero, no cross link will hinder the coil to relax back to its equilibrium conformation although the stretched form of the sample itself is maintained.

The use of quenched samples is of particular interest for structural investigation of non-equilibrium states by SANS, since it uncouples the required measuring time for the scattering experiment from the relaxation times to be investigated. One can measure the whole scattering curve of the same sample at several sample-detector distances which eventually provides all the information available from scattering investigations of non-equilibrium states, i.e. relaxation times at different space scales $[10,11]$. A disadvantage, of course, is the need for the preparation of a separate sample for each time to be investigated. The lower time limit of this method is only given by the time to install the initial non-equilibrium state and to quench the sample to the frozen state. 
The direct measurement of the kinetics of a relaxing system is only possible if the relaxation time is large with respect to the requested measuring time, but the exponential decay of most parameters with the necessity of short scattering runs in the beginning and the possibility of long runs at the end of a kinetic experiment calls for a careful organisation of the allocated beam time. Typical kinetic processes which can readily be followed by SANS approach equilibrium in the range from hours to days (see [12] for the kinetics of $\mathrm{Fe}^{3+}$ hydrolysis in water). A disadvantage of direct kinetic measurement often involves the preparation of a new reacting sample for each instrument configuration (different $Q$-range).

The lower limits for time resolution in direct kinetic measurements are either given by the scattering power of the sample in conjunction with the primary beam intensity or by the limited data acquisition rate of detector and electronics. They have been reached with a stopped-flow apparatus for SANS at D11 [13], which gave for the diffusion of sucrose into the hollow sphere of apoferritin a half time of $\approx 5 \mathrm{~s}$.

\subsection{CyCliC EXPERIMENTS. - Neutron scattering} investigations of phenomena occurring at a time scale shorter than $10 \mathrm{~s}$ are advantageously performed with repeated data collection. Stopped-flow experiments can readily be adopted to a repetitive experimental mode. However, in this mode the consumption of the reacting solutions which goes up with increasing reaction rate can become a limiting factor, especially with SANS experiments, where the relatively large filling volume of $800 \mu \mathrm{l}$ was needed [13]. Small angle $\mathrm{X}$-ray scattering (SAXS) at synchrotron sources, on the other hand, due to the higher intensity of the primary beam, allows the relatively small sample volume of $60 \mu \mathrm{l}$ per cycle with a dead time of $80 \mathrm{~ms}$ for the filling and rinsing of the cell.

A cyclic experiment with practically no dead time and optimal use of the available constant primary beam intensity can be performed with a periodic stretching apparatus, with which the molecular response to an external deformation is measured [15]. The PDMS network described in section 3.1.1 is here subjected to a sinusoidal strain at different frequencies. A marked delay with respect to the corresponding equilibrium values is observed at the higher frequency in figure 7 . The phase shift corresponds roughly to a relaxation time of $\tau=20 \mathrm{~ms}$ at the length scale of the overall size of the labelled chain segments between two cross-link-points. Comparison of this relaxation time with the applied longitudinal flow gradient $\left(G_{\mathrm{L}} \cdot \tau=0.1\right)$ suggests that a larger number of segments interconnected by cross-link-points is stretched by the gradient and the overall deformation of this larger part is transmitted to the segment between the cross link points.

It should be mentioned that this periodic stretching experiment exhibited several experimental peculia-
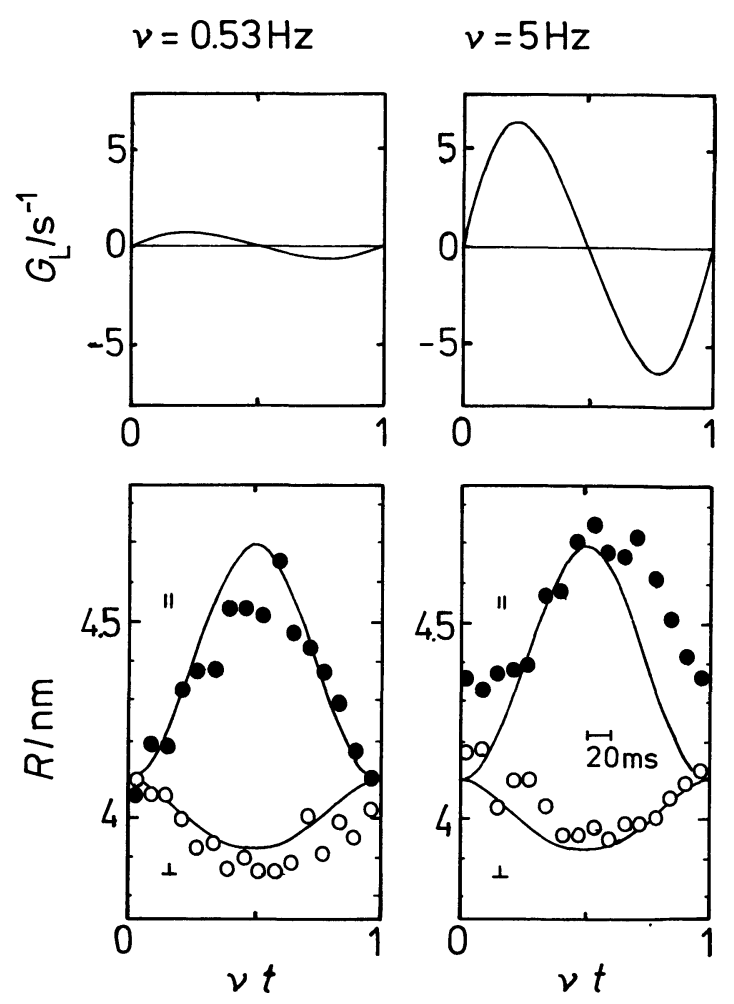

Fig. 7. - Longitudinal flow gradient $G_{\mathrm{L}}$ produced in a sample of rubbery PDMS (cf. Fig. 4) by oscillatory elongation between $1<l / l_{0}<1.5$ at two different frequencies and corresponding relaxation of the mean square radius parallel $(\bullet)$ and perpendicular $(O)$ to the strain with respect to the corresponding calculated equilibrium values for the phantom network (drawn curves) [15].

rities. Not only the monitored scattered intensity,

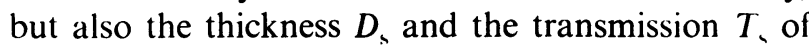
the sample varied within the time period. They had to be measured in a separate cyclic run, where $D(v t)$ could be deduced from $T(v t)$ by

$$
D(v t)=\frac{-1}{\Sigma} \ln T(v t)
$$

and $\Sigma$, the total scattering cross section of the sample was determined in a static experiment. Energy dissipation within the sample was a minor problem due a sufficient cooling by the surrounding air.

3.4 STEAdY STATE EXPERIMENTS. - In both the examples for the relaxation experiment and the cyclic experiment on polymer elongation longitudinal flow gradients occurred, which in the first case served to install non-equilibrium conditions, with $G_{\mathrm{L}} \cdot \tau \gg 1$, which led to an affine deformation of the polymer coil, whereas in the second case, with $G_{\mathbf{L}} \cdot \tau<1$, an increasing delay in the molecular response to the periodic constraint was observed. To maintain such a longitudinal flow gradient at a constant level over a long time period, however, is very difficult.

This is not the case for a transverse or shear gra- 
dient, which can be installed and maintained in a Couette-type scattering cell, made of quartz, which is practically transparent for neutrons and has a negligible small angle scattering [16]. It allows the continuous observation of polymer coils in solution or melt, continuously being deformed in the gradient and continuously trying to relax back towards equilibrium from the imposed deformation (see insert in Fig. 8).

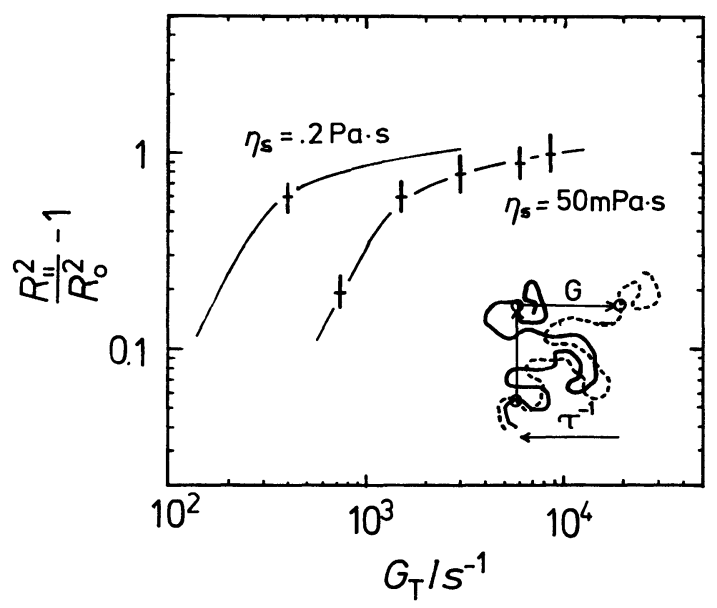

Fig. 8. - Deformation of deuterated PS molecules with $M=3 \times 10^{5} \mathrm{~g} / \mathrm{mole}$ in dilute solution in two viscous solvents (toluene/oligostyrene mixtures) as a function of the applied shear gradient $G_{\mathrm{T}}$. The deformation is expressed by the ratio of the mean square radii of the molecule parallel to the flow direction and at rest. The drawn curves are only guides for the eye and can be superimposed by a horizontal shift.

Such a steady state can only be maintained by a continuous input of energy which is dissipated in the system and which must continuously be removed by heat exchange.

For steady state experiments the time relevant information is given by the rate of deformation, imposed by the sample environment, at which the sample can no more restore its equilibrium conformation by its statistical fluctuations. A marked deformation should occur at $G_{\mathbf{T}} \cdot \tau>1$. Comparison of the slowest Rouse modes for the PS of $M=3 \times 10^{5} \mathrm{~g} / \mathrm{mole}$ at the two different solvent viscosities with the corresponding data on the coil deformation with increasing shear gradient as monitored by the reduced mean square radii in figure 8 confirms this assumption. Hence, the advantage of steady state experiments lies in the fact that the information they provide about relaxation times within the system is not restricted by equations (2) and (3), which are only relevant for single or cyclic non-equilibrium experiments. In these experiments the time relevant information is carried with the scattered neutrons telling the story about the sample they have just met, but in steady state experi- ments the time relevant information is given only by the sample environment (shear rate) and the neutrons encounter always the same average situation at their interaction with the sample.

\section{Outlook}

These few examples from polymer research have already shown the possible variety of SANS experiments under non-equilibrium conditions and their interconnection with other scattering techniques. From figure 3 it is evident, that quasielastic scattering and non-equilibrium SANS are complementary methods providing information in different space/time domains.

For future development in non-equilibrium sample environments a stopped-flow apparatus well adapted for kinetic studies with SANS in the time range below $10 \mathrm{~s}$ would certainly be of great interest especially in molecular biology for the study of the self assembly of large molecules like ribosomes and virusses from specifically labelled subunits. From the polymer and colloid point of view it might be of more interest to develop sample environment with pulsed electrostatic or magnetic fields, cyclic shear or temperature for the study of the kinetics of phase transition or the transition from an ordered to a disordered state.

However, all this does not extend the field of nonequilibrium SANS towards smaller time resolution. It only fills up the space/time field offered by existing or planned neutron scattering instruments and already partly covered by the stretching machine and the shear flow apparatus with further equipment for cyclic nonequilibrium experiments.

The limits of time resolution for cyclic experiments with existing equipment could already now be reached at very low angles if the structural relaxation of very large particles like specifically labelled latex particles of more than $200 \mathrm{~nm}$ diameter in a periodically stretched rubber are to be studied at the same time scale as the coil relaxation in the rubbery PDMS network. If experiments in this space/time domain would become interesting, then a new kind of SANS device especially designed for real time SANS experiments and using thermal or even hot neutrons with a $1 \%$ monochromator and a high resolution detector with $1 \mathrm{~mm}^{2}$ detection elements would extend the limits of time resolution for cyclic experiments by a factor of as much as 100 below the limits now given for D11 with its $10 \%$ selector in the SAS region.

It is interesting to remark that cyclic real time experiments could be extended towards shorter time resolution by the use of short wavelength neutrons whereas the NSE method can be extended towards larger time resolution by the use of longer wavelength neutrons [17], which could eventually lead to an overlap of both techniques. 


\section{References}

[1] Becker, R., Theorie der Wärme (Springer Verlag. [10] BouE, F., Thèse, Université Paris-Sud, 1982. Berlin) 1966.

[2] ReIF, F., Fundamentals of Statistical and Thermal Physics (McGraw Hill Book Co.) 1965.

[3] Landau, L. D., Lifschitz, E. M., Statistical Physics (Pergamon Press, London) 1959.

[4] Neutron Research Facilities at the ILL High Flux Reactor, Institut Laue-Langevin, Grenoble, 1983.

[5] Beltzung, M., Thèse, UlP Strasbourg, 1982.

[6] Pearson, D. S., Macromolecules 10 (1977) 696.

[7] Nicholson, L. K., Higgins, J. S., Hayter, J. B., Macromolecules 14 (1981) 836.

[8] BüLDT, G., Dissertation, Universität Mainz, 1972.

[9] Akcasu, A. Z., Benmouna, M., Han, C. C., Polymer $21(1980) 866$.

[11] Boue, F., Nierlich, M., JanNinK, G., Ball, R., J. Physique 43 (1982) 137.

[12] Christensen, A. N., Lehmann, M. S., Wright, A., Acta Chem. Scand. A 37 (1983) 63.

[13] HaAs, J. A., Dissertation, Universität Mainz, 1977.

[14] Berthet-Colominas, C., Bois, J. M., Cuillel, M., Sedita, J., Vachette, P., Revue Phys. Appl., this issue.

[15] Rennie, A. R., Oberthür, R. C., Revue Phys. Appl., this issue.

[16] Lindner, P., Oberthür, R. C., Revue Phys. Appl., this issue.

[17] MezeI, F., in The Installation of a Second Cold Source, ILL Internal Report, Grenoble, 1982. 